\title{
A comparative study of the sarcopenia screening in older patients with interstitial lung disease
}

\author{
Masatoshi Hanada ${ }^{1,2^{*}}$, Noriho Sakamoto ${ }^{3}$, Hiroshi Ishimoto ${ }^{3}$, Takashi Kido ${ }^{3}$, Takuto Miyamura ${ }^{3}$, \\ Masato Oikawa ${ }^{1,2}$, Hiroki Nagura ${ }^{1,2}$, Rina Takeuchi ${ }^{1}$, Yurika Kawazoe ${ }^{4}$, Shuntaro Sato ${ }^{4}$, S. Ahmed Hassan ${ }^{5}$, \\ Yuji Ishimatsu', Hideaki Takahata', Hiroshi Mukae ${ }^{3}$ and Ryo Kozu ${ }^{1,2}$
}

\begin{abstract}
Background: The Asian Working Group for Sarcopenia 2019 (AWGS 2019) is the gold standard diagnostic criteria for sarcopenia in Asian populations. The calf circumference (CalF), the strength, assistance in walking, rising from a chair, climbing stairs, and falls (SARC-F) and the SARC-CalF questionnaires for sarcopenia screening have been used by AWGS 2019. The aim of this study was to assess accuracy of these three sarcopenia screening tools in patients with interstitial lung disease.

Methods: In this cross-sectional study, stable patients with interstitial lung disease were enrolled. The SARC-F, SARCCalF, and CalF, used in patients with interstitial lung disease, were compared to the diagnostic criteria proposed by AWGS 2019. The accuracy of screening tools was compared using sensitivity and specificity. Moreover, areas under the receiver operating characteristic curves (AUC) were computed.

Results: Seventy eight patients were analyzed, and sarcopenia was identified in 25 (32.1\%) patients with interstitial lung disease by the AWGS 2019 criteria. The sensitivity of the CalF was highest (96\%) of the three screening tools, while the specificity was $60 \%$. The sensitivity of SARC-F and SARC-CalF were $24 \%$ and $68 \%$, while the specificity were $92 \%$ and $66 \%$, respectively. The AUCs of CaIF, SARC-F, and SARC-CalF in all patients were $0.78,0.58$, and 0.67, respectively.
\end{abstract}

Conclusions: The CalF is most suitable for screening sarcopenia in patients with interstitial lung disease, while SARCF and SARC-CalF are not.

Keyword: Interstitial lung diseases, Physical performance, Sarcopenia, Frailty

\section{Background}

Aging is associated with increased susceptibility to a variety of chronic diseases including lung pathologies, such as ILD [1]. Milne et al. have pointed out that frailty is highly prevalent in ILD patients [2]. Frailty and

\footnotetext{
*Correspondence: mstshnd@nagasaki-u.ac.jp

1 Department of Rehabilitation Medicine, Nagasaki University Hospital,

1-7-1 Sakamoto, Nagasaki 852-8501, Japan

Full list of author information is available at the end of the article
}

sarcopenia result in a loss of functional independence, where sarcopenia may be a risk factor for frailty $[3,4]$. Sarcopenia has been defined as a progressive and generalized skeletal muscle disorder that involves the accelerated loss of muscle mass and function, and increased adverse outcomes including falls, functional decline, frailty, and mortality $[5,6]$. The relationship between sarcopenia and adverse outcomes has been reported in various clinical populations [7-9]. In brief, sarcopenia is directly linked to activity limitation and is a huge concern 
for older patients. Despite knowledge of these risk factors, evaluation of sarcopenia is not common and little is known about its epidemiology in older patients with ILD [10].

Sarcopenia diagnosis and treatment were defined for Asians in the Asian Working Group for Sarcopenia (AWGS) 2014 consensus, and updated by the AWGS 2019 consensus [11]. In AWGS 2019, either calf circumference (CalF), the strength, assistance in walking, rising from a chair, climbing stairs, and falls (SARC-F) questionnaire or SARC-F combined with CalF (SARC-CalF) questionnaire were utilized for case finding.

The SARC-F was proposed in 2013, and is regarded as a simple questionnaire [12]. This questionnaire is also used in case finding in European Working Group on Sarcopenia in Older People (EWGSOP) 2 [5]. This questionnaire offers high specificity to diagnose sarcopenia, but poses the issue of low sensitivity. Moreover, several studies have pointed out that the SARC-F underestimates prevalence $[13,14]$, which, however, can be measured more accurately with the SARC-CalF that adds a measure of CalF to SARC-F [15]. Important to note, ILD patients experience dyspnea with exertion and SARC-F that includes factors such as activity limitation through exertion may not be suitable for screening of chronic respiratory diseases including ILD. The most suitable choice to evaluate sarcopenia for chronic respiratory diseases with dyspnea between SARC-F, the SARC-CalF and CalF is unknown.

The present study aimed to assess physical performance screening tools for sarcopenia in patients with ILD including the SARC-F, SARC-CalF and CalF in comparison to the diagnostic criteria proposed by the AWGS 2019. Additionally, we aimed to determine the most effective tool to assess sarcopenia.

\section{Methods}

\section{Study design}

This was a prospective, cross-sectional observational study that enrolled ILD patients $\geq 60$ years of age from January 2020 to May 2021. Subjects gave their written, informed consent, and the study was approved by the Human Ethics Review Committee of Nagasaki University Hospital (approval number: 19121610).

\section{Subjects}

Patients with ILD, including idiopathic interstitial pneumonias (IIPs), connective tissue disease-associated interstitial pneumonia, and hypersensitivity pneumonitis were recruited at the Department of Respiratory Medicine, Nagasaki University Hospital. Diagnostic criteria for IIPs and hypersensitivity pneumonitis were consistent with the International Consensus Statement [16]. Subjects were included if they were under the care of a respiratory physician, were ambulant, and were clinically stable with no changes in medication for at least four weeks before enrollment. Exclusion criteria were comorbid conditions affecting exercise performance (e.g., musculoskeletal or neurological disorders,), severe cognitive impairment, pregnancy, recent thoracic surgery, and active cancer treatment.

\section{Measurement \\ ILD-GAP model}

The interstitial lung disease-gender, age and lung physiology (ILD-GAP) model was created by adding the ILD subtype variable to the original GAP model [17]. The two lung physiology variables in this model include forced vital capacity (FVC) and diffusion capacity for carbon monoxide (DLco). Points were assigned for each variable to obtain a total point score (range 0-8). Demographic and clinical information including physical function, biochemistry of blood and pulmonary function test results were obtained from medical records. Subjects were divided into two groups: low ILD-GAP group (ILD GAP score 0-2) and high ILD-GAP group (ILD GAP score $3-8)[18,19]$. The results of the three screening methods were compared in each group.

\section{The SARC-F Questionnaire, the SARC-CalF Questionnaire, and CalF}

The SARC-F Questionnaire was used to measure probable sarcopenia. The SARC-F questionnaire for sarcopenia is shown in Fig. 1 [12]. This questionnaire is composed of five items: strength, assistance in walking, rising from a chair, climbing stairs, and falls [12]. The SARC-F scores range from 0 to 10 , with 0 to 2 points for each component [scoring range: 0 (best) to 10 (worst)]. Patients with a total score $\geq 4$ were classified as having a risk of sarcopenia [20]. The SARC-CalF questionnaire is a combination of calf circumference and SARC-F, and a score of $\geq 11$ points indicates probable sarcopenia. The maximum CalF was measured in a sitting position with hip and knee joint flexed at approximately $90^{\circ}$. CalF was measured at the point of the largest circumference. Calf $<34 \mathrm{~cm}$ for males and $<33 \mathrm{~cm}$ for females indicated sarcopenia. The CalF item is scored as 0 points if the CalF is $>34 \mathrm{~cm}$ for males and $>33 \mathrm{~cm}$ for females and 10 points if the calf circumference is $\leq 34 \mathrm{~cm}$ for males and $\leq 33 \mathrm{~cm}$ for females [15].

\section{Diagnostic criteria for sarcopenia}

Since the participants were older Japanese patients, they were evaluated according to the AWGS 2019 criteria of sarcopenia [11]. The diagnostic criteria for sarcopenia as used in AWGS 2019 is shown in Fig. 2. Estimation of sarcopenia at the first stage requires measurement by three 


\begin{tabular}{lll}
\hline Component & Question & Scoring \\
\hline Strength & How much difficulty do & None $=0$ \\
& you have in lifting and & Some $=1$ \\
& carrying 10 pounds? & A lot or unable = 2
\end{tabular}

$\begin{array}{lll}\text { Assistance in walking } & \begin{array}{l}\text { How much difficulty do } \\ \text { you have walking across a } \\ \text { room? }\end{array} & \begin{array}{l}\text { None }=0 \\ \text { Some }=1 \\ \text { A lot, use aids, or } \\ \text { unable }=2\end{array} \\ \text { Rise from a chair } & \begin{array}{l}\text { How much difficulty do } \\ \text { you have transferring } \\ \text { from a chair or bed? }\end{array} & \begin{array}{l}\text { None }=0 \\ \text { Some }=1\end{array} \\ & \begin{array}{l}\text { A lot or unable without } \\ \text { help }=2\end{array} \\ \text { Climb stairs } & \begin{array}{ll}\text { How much difficulty do } \\ \text { you have climbing a flight } \\ \text { of } 10 \text { stairs? }\end{array} & \begin{array}{l}\text { None }=0 \\ \text { Some }=1\end{array} \\ \text { Falls } & \begin{array}{l}\text { A lot or unable }=2 \\ \text { fallen in the past year? }\end{array} & \begin{array}{l}1-3 \text { falls }=1 \\ 4 \text { or more falls }=2\end{array}\end{array}$

Fig. 1 The SARC-F questionnaire for sarcopenia. The SARC-F: the strength, assistance in walking, rising from a chair, climbing stairs, and falls

screening methods: CalF, SARC-F and SARC-CalF. The second stage involves measurement of gait speed, grip strength, and muscle mass. Based on the AWGS 2019, low muscle strength is defined as handgrip strength $<28$ $\mathrm{kgf}$ for males and $<18 \mathrm{kgf}$ for females, while low physical performance is characterized as gait speed $<1.0 \mathrm{~m} / \mathrm{s}$. In addition, body composition and skeletal muscle mass were evaluated in ILD patients using the bioelectrical impedance analysis (BIA) method (InBody 270, InBody Japan, Tokyo, Japan). Low muscle mass diagnosis was defined as skeletal muscle index $(\mathrm{SMI})<7.0 \mathrm{~kg} / \mathrm{m}^{2}$ for males and $<5.7 \mathrm{~kg} / \mathrm{m}^{2}$ for females.

\section{Assessment of peripheral muscle force}

Peripheral muscle force was evaluated via measurements of quadriceps and handgrip forces. Peak force developed during a maximal isometric knee extension was used as a measure of quadriceps force (QF). A hand-held dynamometer with a fixing belt ( $\mu$-Tas F-1; Anima Corporation, Tokyo, Japan) was used following a standard protocol [21]. The QF for the dominant side was tested in the sitting position with the hip and knee joints flexed at approximately $90^{\circ}$. The handgrip force (HF) was assessed in the dominant hand using a dynamometer (T.K.K.5401; Takei-Kiki-Kogyou Corporation, Niigata, Japan). HF was tested in sitting position with the elbow flexed at $90^{\circ}$ and the arms fixed to the body. The average value of three attempts was recorded.

\section{The short physical performance battery (SPPB)}

Physical performance was measured using the SPPB [22], which consists of three measures: walking speed, chair stands, and standing balance. A score from 0 (unable to complete the task) to 4 (best performance possible) was assigned to each measurement. The total score $(0-12)$ was used to estimate overall physical performance level. Similar to previous studies [23-25], patients were separated into low physical performance-related risk $(\mathrm{SPPB} \leq 9)$ or high physical performance-related risk $(\mathrm{SPPB}>9)$.

\section{Functional exercise capacity}

The 6-min walk test was performed based on published guidelines [26, 27]. The greater distance of two attempts 


\section{Acute to chronic health care or clinical research settings}

\section{Case finding}

Presence of any of the following clinical conditions:

- Functional decline or limitation; unintentional weight loss; depressive mood; cognitive impairment; repeated falls; malnutrition

- Chronic conditions (heart failure, chronic obstructive pulmonary disease, diabetes mellitus, chronic kidney disease, etc.)

If no clinical conditions above are present:

- Calf circumference (male: $<34 \mathrm{~cm}$, female: $<33 \mathrm{~cm}$ ) or SARC-F $\geq 4$ or SARC-CalF $\geq 11$

\section{Diagnosis}

Muscle strength

- Handgrip strength (male: $<28 \mathrm{~kg}$, female: $<18 \mathrm{~kg}$ )<smiles>[3H][13CH3]</smiles>

Physical performance

- 6-meter walk: $<1.0 \mathrm{~m} / \mathrm{s}$ or 5 -time chair stand test: $\geq 12 \mathrm{~s}$ or SPPB: $\leq 9$ points<smiles>[Tl]</smiles>

Appendicular skeletal muscle mass (ASM)

- Dual-energy $X$-ray absorptiometry (male: $<7.0 \mathrm{~kg} / \mathrm{m}^{2}$, female: $<5.4 \mathrm{~kg} / \mathrm{m}^{2}$ ) or Bioelectrical impedance analysis (male: $<7.0 \mathrm{~kg} / \mathrm{m}^{2}$, female: $<5.7 \mathrm{~kg} / \mathrm{m}^{2}$ )

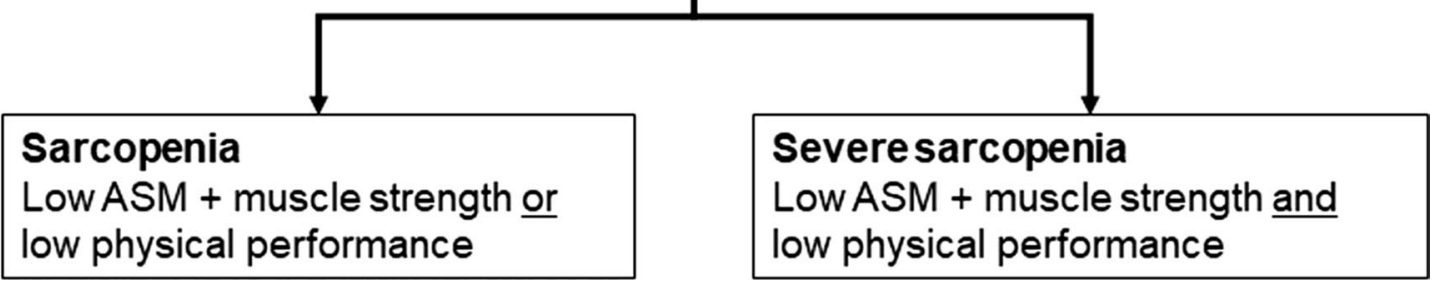

Fig. 2 The diagnostic criteria for sarcopenia used by the Asian Working Group for Sarcopenia 2019. The SARC-F: the strength, assistance in walking, rising from a chair, climbing stairs, and falls; the SARC-CalF: the SARC-F combined with calf circumference; SPPB: short physical performance battery

was recorded. Oxygen saturation $\left(\mathrm{SpO}_{2}\right)$ was monitored continuously throughout both tests (Konica Minolta Pulsox Me Oximeter, Osaka, Japan). If $\mathrm{SpO}_{2}$ decreased below $80 \%$, the tests were terminated. Pre-exercise $\mathrm{SpO}_{2}$ and the lowest $\mathrm{SpO}_{2}$ during the tests were recorded.

\section{Activities of daily living}

Activities of daily living (ADL) were evaluated using the Kats basic ADL scale [28]. The scale evaluates activities including feeding, ability to transfer, dressing, bathing, shopping and transportation. For each of the 6 activities, a score of 0 (dependent) or 1 (independent) was assigned. The sum of scores was used to obtain a measure of ADL performance.

\section{Health-related quality of life}

Health-related quality of life (HRQL) was evaluated using the King's Brief Interstitial Lung Disease (K-BILD) health status Japanese version questionnaire [29]. The K-BILD is an ILD-specific HRQL questionnaire that measures health impairment. It is comprised of 15 items in three domains: breathlessness and activities, chest symptoms, 
and a psychological health. The K-BILD score ranged from 0 to 100 , where higher values indicated better health.

\section{Statistical analysis}

Baseline characteristics were summarized with frequencies and percentages for categorical data, while means and standard deviations for continuous data. The Shapiro-Wilk test was used to examine data distribution. Comparisons between sarcopenia and non-sarcopenia groups were made with unpaired $t$-tests, Mann-Whitney U tests, or Fisher's exact test. To evaluate the accuracy of screening by SARC-F, the SARC-CalF, and CalF, sensitivity, specificity, positive predictive value $(\mathrm{PV}+)$ and negative predictive value (PV-) were calculated for each screening method. The gold standard for calculating sensitivity and specificity was set at AWGS 2019 criteria. ILD-GAP model was used to evaluate the applicability of the three screening methods to ILD patients. To compare the accuracy among the screening tools, receiver operating characteristic curves (ROC) were constructed and area under ROC curves (AUC) were calculated. In addition, Spearman's rank correlation coefficient was used to examine relationship of K-BILD with patient-reported outcomes (SARC-F, SARC-CalF and CalF). The level of significance was 0.05 for all statistical tests. All statistical analyses were performed using JMP 15.0 software (SAS Institute Japan, Tokyo, Japan).

\section{Results}

\section{Patients' characteristics}

The baseline characteristics of the 78 subjects are presented in Table 1 . The mean age of patients was approximately 71 years. In the sarcopenia and non-sarcopenia group, patients had diagnoses of idiopathic pulmonary fibrosis $(n=10, n=31)$, IIPs other than IPF (sarcopenia group $n=12$ : crypto-genic organizing pneumonia $n=3$, pleuroparenchymal fibroelastosis $n=2$, Unclassifiable $\mathrm{n}=7$, non-sarcopenia group $\mathrm{n}=12$ : nonspecific interstitial pneumonia $n=1$, lymphoid interstitial pneumonia $\mathrm{n}=1$, combined pulmonary fibrosis and emphysema $\mathrm{n}=1$, interstitial pneumonia with autoimmune features $n=2$, Unclassifiable $n=6$ ), connective tissue diseaseassociated interstitial pneumonia $(n=9$ : Sjogren syndrome $\mathrm{n}=3$, Mixed Connective Tissue Disease $\mathrm{n}=1$, Rheumatoid arthritis $n=3$, pleuroparenchymal fibroelastosis $n=2)$, and hypersensitivity pneumonitis $(n=2$, $n=2$ ), respectively. The number of patients treated corticosteroid was not significantly different between sarcopenia group and non-sarcopenia group (Table 1). Furthermore, the duration (median (IQR)) of diagnosis to enrollment in patients receiving corticosteroid in sarcopenia group was $2(1-14)$ months and 3 (1-18) months in the non-sarcopenia group. These results were not significantly different between groups. Type of ILD patients receiving corticosteroid were CHP $(n=2)$, CVD-IP $(\mathrm{n}=4), \operatorname{COP}(\mathrm{n}=2)$ and unclassified $(\mathrm{n}=4)$. Pulmonary function tests were similar in both groups. The HF and QF as peripheral muscle force were not significantly different between groups with and without sarcopenia. The CalF, SMI, SARC-F and the SARC-CalF were not significantly different between sarcopenia and non-sarcopenia groups, separately. All patients were independent according to the ADL score. In HRQL, the total score of K-BILD questionnaire was within the average range $50-60 \%$ for both groups.

\section{Prevalence of sarcopenia}

In our patients, the prevalence of sarcopenia was $32.1 \%$ according to the AWGS 2019 criteria: males: 31.4\%; females: $33.3 \%$. There were no significant differences in the prevalence of sarcopenia between the two sexes. Overall, 24 (96\%) patients screened positive for sarcopenia using CalF, 6 (24\%) using SARC-F questionnaire, and 17 (68\%) using SARC-CalF questionnaire based on the AWGS 2019 criteria. Significant negative correlations were found between K-BILD and SARC-F $(\mathrm{r}=-0.243$, $p=0.033)$, but not SARC-CalF $(\mathrm{r}=-0.128, p=0.267)$ or CalF $(r=0.137, p=0.233)$.

\section{Sensitivity, specificity and predictive values of sarcopenia screening}

The sensitivity of the SARC-F for the detection of case finding in sarcopenia was 24\%; conversely, the specificity was $92 \%$ (Table 2). Overall, the sensitivity of the CalF was 96\%, the highest amongst the three sarcopenia screening tools. The PV + of all items were low whereas the PV- were relatively high $(>50 \%)$. The results of the sensitivity-specificity analysis were not affected by high or low ILD-GAP score groups (ILD severity). Furthermore, CalF provided better sensitivity than other items of SARC-F and SARC-CalF in patients with ILD (Table 3). The CalF alone had the best sensitivity (AUC) in screening sarcopenia compared to SARC-F and SARC-CalF according to AWGS 2019 criteria (Fig. 3).

\section{Discussion}

The main findings of the present study are as follows: (1) in ILD patients with normal BMI and mild mMRC dyspnea scale, sarcopenia was identified in 25 (32.1\%) patients by the AWGS 2019 criteria; (2) the CalF was the most sensitive in identifying sarcopenia compared to SARC-F and SARC-CalF. These screening tools showed a similar trend regardless of the ILD-GAP score (severity of ILD); (3) CalF items had better sensitivity in the SARC-CalF. To our knowledge, this is the first report comparing the 
Table 1 Patients' characteristics

\begin{tabular}{|c|c|c|c|c|}
\hline & \multirow{2}{*}{$\begin{array}{l}\text { Overall } \\
(n=78)\end{array}$} & \multicolumn{3}{|c|}{ AWGS 2019 criteria classification } \\
\hline & & $\begin{array}{l}\text { Sarcopenia } \\
(n=25)\end{array}$ & $\begin{array}{l}\text { Non-sarcopenia } \\
(n=53)\end{array}$ & $P$ value \\
\hline Age, years & $71(67-77)$ & $72(68-77)$ & $71(67-82)$ & 0.499 \\
\hline Sex male, \% & $51(65)$ & $16(64)$ & $35(66)$ & 1.00 \\
\hline $\mathrm{BMl}, \mathrm{kg} / \mathrm{m}^{2}$ & $24(20-26)$ & $23(20-28)$ & $24(22-26)$ & 0.855 \\
\hline Diagnosis, IIPs/other ILDs & $49 / 29$ & $15 / 10$ & $34 / 19$ & 0.804 \\
\hline Duration from diagnosis, months & $3(1-16)$ & $2(1-12)$ & $6(1-23)$ & 0.227 \\
\hline Corticosteroid, $\%$ & $12(15)$ & $6(24)$ & $6(11)$ & 0.184 \\
\hline Antifibrotic drugs, $\%$ & $16(21)$ & $4(16)$ & $12(23)$ & 0.564 \\
\hline Long-term oxygen therapy, \% & $11(14)$ & $5(20)$ & $6(11)$ & 0.316 \\
\hline ILD-GAP score, point & $3(2-4)$ & $3(2-4)$ & $3(2-4)$ & 0.551 \\
\hline mMRC dyspnea scale, grade & $2(1-2)$ & $2(1-2)$ & $1(1-2)$ & 0.754 \\
\hline $\mathrm{PaCO}_{2}$ at rest, $\mathrm{mmHg}$ & $41(38-46)$ & $40(38-44)$ & $42(38-51)$ & 0.417 \\
\hline $\mathrm{PaO}_{2}$ at rest, $\mathrm{mmHg}$ & $80(63-91)$ & $86(73-94)$ & $75(61-91)$ & 0.219 \\
\hline $\mathrm{FVC}, \%$ & $85(68-97)$ & $85(63-93)$ & $85(72-98)$ & 0.316 \\
\hline $\mathrm{FEV}_{1}$, \%pred & $90(75-101)$ & $90(69-103)$ & $90(77-101)$ & 0.863 \\
\hline $\mathrm{FEV}_{1} / \mathrm{FVC}, \%$ & $78(72-86)$ & $80(72-87)$ & $78(72-85)$ & 0.514 \\
\hline $\mathrm{DL}_{\mathrm{CO},} \%$ & $64(45-80)$ & $70(45-83)$ & $60(45-80)$ & 0.271 \\
\hline $\mathrm{KL}-6, \mathrm{U} / \mathrm{ml}$ & $715(451-1165)$ & $662(474-1168)$ & 740 (407-1155) & 0.768 \\
\hline $\mathrm{HF}, \mathrm{kgf}$ & $26(20-33)$ & $23(19-33)$ & $27(22-34)$ & 0.167 \\
\hline QF, kgf & $28(19-35)$ & $23(16-33)$ & $29(20-36)$ & 0.169 \\
\hline Calf circumference, $\mathrm{cm}$ & $33(30-35)$ & $33(30-37)$ & $34(30-35)$ & 0.996 \\
\hline $\mathrm{SMI}, \mathrm{kg} / \mathrm{m}^{2}$ & $7(6-7)$ & $7(5-7)$ & $7(6-8)$ & 0.201 \\
\hline SPPB, point & $11(11-12)$ & $12(11-12)$ & $12(11-12)$ & 0.912 \\
\hline SARC-F, point & $1(0-2)$ & $1(0-4)$ & $1(0-2)$ & 0.486 \\
\hline SARC-CalF, point & $2(0-11)$ & $11(0-12)$ & $1(0-11)$ & 0.171 \\
\hline 6MWD, m & $424(355-500)$ & $392(308-509)$ & $442(371-500)$ & 0.317 \\
\hline ADL score & $6(6-6)$ & $6(6-6)$ & $6(6-6)$ & 0.204 \\
\hline K-BILD, point & $54(47-64)$ & $53(44-68)$ & $54(48-62)$ & 0.684 \\
\hline Psychological symptoms & $51(43-63)$ & $49(43-67)$ & $52(42-60)$ & 0.720 \\
\hline Breathlessness and activities & $44(34-53)$ & $40(31-55)$ & $46(37-53)$ & 0.232 \\
\hline Chest symptoms & $73(64-85)$ & $73(47-85)$ & $73(64-85)$ & 0.779 \\
\hline
\end{tabular}

Categorical variables were analyzed using Fisher's exact test, while continuous variables with unpaired t-tests and Mann-Whitney $\mathrm{U}$ test. Values are median (interquartile range) or numbers (percentage) of subjects

ADL score, activities of daily living score; BMI, Body Mass Index; CalF, calf circumference; DLCO, diffusion capacity for carbon monoxide; \%FEV1, percentage of forced expiratory volume in one second; FVC, forced vital capacity; HF, handgrip force; IIPs, idiopathic interstitial pneumonias; ILD-GAP, interstitial lung disease-gender, age, lung physiology score; K-BILD, King's Brief Interstitial Lung Disease, KL-6, Krebs von den Lungen-6; mMRC, Modified Medical Research Council; 6MWD, six-minute walking distance; $\mathrm{PaCO}$, partial pressure of carbon dioxide; $\mathrm{PaO} 2$, partial pressure of oxygen; \%pred, percent predicted; $\mathrm{QF}$, quadriceps force; the SARC-F, the strength, assistance in walking, rising from a chair, climbing stairs, and falls; SARC-CalF, SARC-F combined with calf circumference; SMI, skeletal muscle index; SPPB, short physical performance battery; \%VC, percentage of volume capacity

accuracy of SARC-F, SARC-CalF, and CalF in screening sarcopenia in patients with ILD.

Sarcopenia was identified in 25 (32.1\%) patients with ILD by the AWGS 2019 criteria. Although, previous studies have reported the sarcopenia prevalence of $5-25 \%$ in older population [11,30-32], the present study indicated a higher prevalence. This highlights the importance of accurate sarcopenia screening to improve patient outcomes. Improvement in survival rate is expected in ILD patients if their disease progression is suppressed by treatment (e.g., antifibrotic drugs). However, it is speculated that aging associated sarcopenia may pose a problem [33]. Therefore, it is important to screen sarcopenia in patients with ILD as early as possible.

In the present study, the SARC-F had low sensitivity, high specificity, low $\mathrm{PV}+$ and high $\mathrm{PV}-$. These results are consistent with those in different populations [34-36]. The poor sensitivity reported in this study and several 
Table 2 Sensitivity, specificity and predictive values of three sarcopenia screening in patients with ILD

\begin{tabular}{lllll}
\hline & Sensitivity (\%) & Specificity (\%) & PV+ & PV- \\
\hline $\begin{array}{lllll}\text { All patients } \\
\quad\end{array}$ Calf circumference & 96.0 & 60.0 & 53.3 & 97.0 \\
SARC-F & 24.0 & 92.0 & 60.0 & 72.1 \\
$\quad$ SARC-CalF & 68.0 & 66.0 & 48.6 & 81.4 \\
Low ILD-GAP score <3 & & & & \\
$\quad$ Calf circumference & 100 & 41.0 & 47.4 & 100 \\
SARC-F & 11.1 & 94.0 & 50.0 & 66.7 \\
SARC-CalF & 77.8 & 53.0 & 46.7 & 81.8 \\
High ILD-GAP score $\geq 3$ & & & & \\
$\quad$ Calf circumference & 91.7 & 72.0 & 57.9 & 95.5 \\
SARC-F & 33.3 & 93.0 & 66.7 & 77.1 \\
SARC-CalF & 58.3 & 72.0 & 46.7 & 80.8 \\
\hline
\end{tabular}

ILD, interstitial lung disease; ILD-GAP, interstitial lung disease-gender, age, lung physiology score; $\mathrm{PV}+$, positive predictive value, $\mathrm{PV}$-, negative predictive value; SARC-F, the strength, assistance in walking, rising from a chair, climbing stairs, and falls; SARC-CalF, SARC-F combined with calf circumference previous studies may suggest that SARC-F is not a suitable screening test for sarcopenia in ILD patients. The issue of underestimation of accuracy by SARC-F was resolved using SARC-CalF that adds a measure of CalF to SARC-F [13]. Mo et al. pointed out that low-to-moderate sensitivity of the SARC-F is not adequate for population wide screening [30]. Similar conclusion was drawn in this study with a small, yet specific group of ILD patients. Moreover, almost all questions in the SARC-F and SARCCalF are affected by dyspnea through exertion except CalF. Therefore, it was speculated that the SARC-F and SARC-CalF are not suitable for screening sarcopenia in patients with chronic respiratory diseases including ILD. However, the SARC-F had the highest specificity in our results. Although the SARC-F may be an effective tool for selecting subjects who should undergo further testing for sarcopenia, careful attention should be paid to the fact that there could be multiple omissions when SARC$\mathrm{F}$ is used for excluding sarcopenia [37]. In this study, we

Table 3 Sensitivity, specificity and predictive values of items of the SARC-F and calf circumference in screening for sarcopenia in patients with ILD

\begin{tabular}{|c|c|c|c|c|c|}
\hline SARC-CalF & $\begin{array}{l}\text { Positive screening } \\
\text { n (\%) }\end{array}$ & Sensitivity (\%) & Specificity (\%) & PV+ & PV- \\
\hline Strength & $29(34.5)$ & 33.3 & 69.0 & 36.0 & 66.0 \\
\hline Assistance in walking & $8(9.5)$ & 71.4 & 72.0 & 20.0 & 96.2 \\
\hline Rising from a chair & $11(13.1)$ & 44.4 & 70.0 & 16.0 & 90.1 \\
\hline Climbing stairs & $48(57.1)$ & 34.1 & 71.0 & 60.0 & 45.3 \\
\hline Falls & $16(19.0)$ & 33.3 & 68.0 & 20.0 & 81.1 \\
\hline Calf circumference & $48(57.1)$ & 100 & 51.0 & 50.0 & 100 \\
\hline
\end{tabular}

ILD, interstitial lung disease; $P V+$, positive predictive value, $P V-$, negative predictive value; SARC- $F$, strength, assistance in walking, rising from a chair, climbing stairs, and falls; SARC-CalF, the SARC-F combined with calf circumference
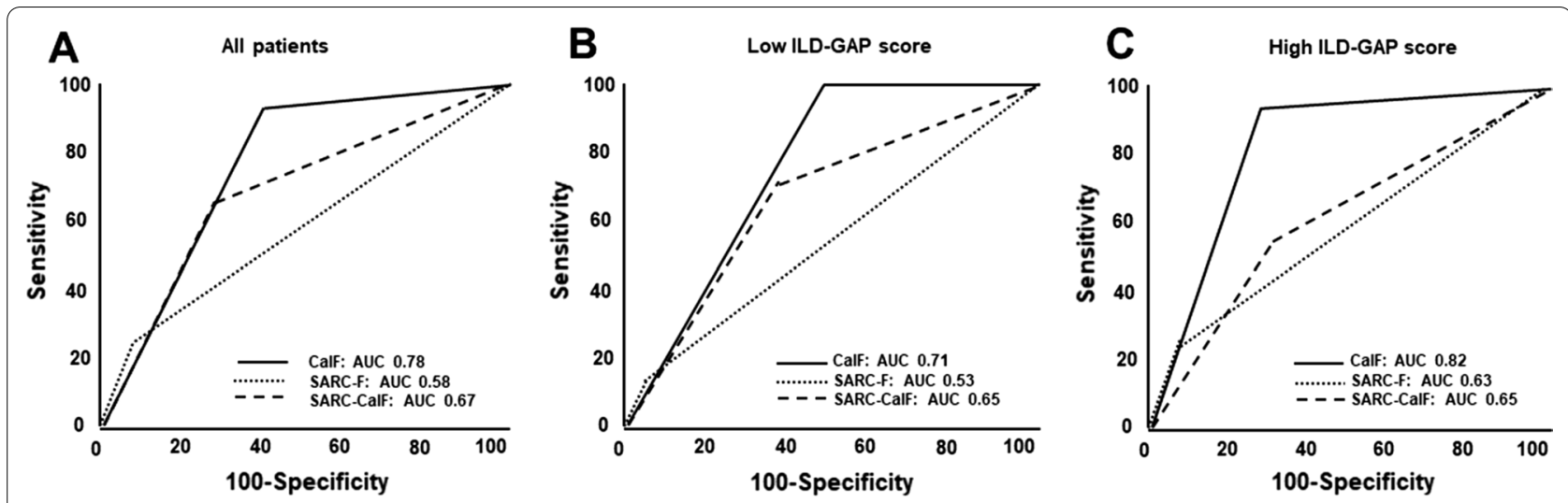

Fig. 3 The ROC curves of three screening methods according to AWGS 2019. A All patients, B low ILD-GAP score group, C high ILD-GAP score group. AUC, area under the curve; CalF, calf circumference; ROC, receiver operating characteristic; the SARC-F, the strength, assistance in walking, rising from a chair, climbing stairs, and falls; the SARC-CalF, the SARC-F combined with calf circumference 
found significant negative correlation between K-BILD and SARC-F. Thus, although SARC-F may not be suitable for excluding sarcopenia, it has the potential to be a useful assessment tool to evaluate health-related quality of life. It should also be noted that in patients with respiratory diseases, the results from questionnaire are likely impacted by age and exertion on dyspnea, making it difficult to assess sarcopenia accurately. In any case, the reliability, validity and utility of SARC-F needs further investigation in patients with respiratory diseases. Conversely, the CalF measurement may be the best screening option as it is simple and convenient. The CalF takes only takes a few minutes to complete, requires little training to administer. In addition, the results can be quantified, are reproducible and sensitive to changes in functionality through time. Therefore, if we can diagnosis only by the CalF measurements to sarcopenia diagnosis, there are considerable benefits for ILD patients, it can be effectively used for early evaluation of sarcopenia in ILD patients. Better screening tools for sarcopenia would help identify frail patients and, thus, prompt more frequent referrals for pulmonary rehabilitation leading to healthrelated quality of life and exercise tolerance.

In SARC-CalF, CalF had better sensitivity than other items. Dyspnea during exertion is characteristic of chronic respiratory diseases including ILD. In this study, unlike previous studies that examined SARC-F items, the positive screening rate was highest for climbing stairs (57.1\%) among the items that involve exertion [31]. The positive screening rate was high, but the sensitivity was low, making it difficult accurately screen for sarcopenia in ILD patients with only stair climbing component. Based on the results of this study, SARC-F and SARCCalF are not suitable screening tool to assess sarcopenia in ILD patients. Therefore, development of a new sarcopenia screening tool for chronic respiratory diseases is desirable.

This study has several limitations. Firstly, the sample size was small and the study was a single-center trial. Larger, multicenter studies are needed to consider the development of a new sarcopenia screening tool. Secondly, in ILD patients excluding IPF, corticosteroids are one of the most common treatment options employed. However, corticosteroids decrease skeletal muscle function in ILD patients with mild symptoms [38] and are an independent risk factor for developing sarcopenia [39]. This suggests that the risk of developing sarcopenia is higher in ILD patients commonly treated by corticosteroids. Since our patients had numerous ILDs for which steroids are not prescribed, relationship between corticosteroid use and sarcopenia could not be evaluated using multivariable analysis. Larger studies are needed to consider the influence of corticosteroid use on developing sarcopenia in ILD patients. Thirdly, when using the SARC-F and the SARC-CalF to screen for sarcopenia according to the AWGS 2019 criteria, it is necessary to adjust the cut-off values that are suitable for chronic respiratory diseases. Finally, we used BIA for the assessment of SMI instead of CT, MRI or DEXA. Although, these methods are more precise, BIA prevents exposure to $\mathrm{x}$-rays and provides a more efficient solution to measuring body composition and skeletal muscle mass. As a result, the AWGS 2019 criteria recommends BIA as an alternative option for muscle measurement.

\section{Conclusion}

In conclusion, approximately $30 \%$ patients with ILD were found to have sarcopenia based on the AWGS 2019 criteria. Calf circumference had the best sensitivity out of the three screening tools and is, thus, most suitable for screening sarcopenia in patients with ILD. In the future, development of a new sarcopenia screening tool for chronic respiratory diseases should be considered.

\begin{abstract}
Abbreviations
ADL: Activities of daily living; AUC: Area under receiver operating characteristic curves; AWGS: The Asian Working Group for Sarcopenia; BIA: Bioelectrical impedance analysis; BMI: Body Mass Index; CalF: Calf circumference; $\mathrm{DL}_{\mathrm{CO}}$ : Diffusion capacity for carbon monoxide; EWGSOP: European Working Group

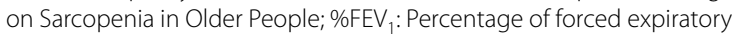
volume in one second; FEV $/$ /FVC: Forced expiratory volume in one second/ forced vital capacity; HF: Handgrip force; HRQL: Health-related quality of life; IIPs: Idiopathic interstitial pneumonias; ILD: Interstitial lung disease; ILD-GAP model: Interstitial lung disease-gender, age, lung physiology; IPF: Idiopathic pulmonary fibrosis; K-BILD: King's Brief Interstitial Lung Disease; mMRC dyspnea: Modified Medical Research Council dyspnea; 6MWD: 6-Minute walking distance; $\mathrm{PaCO}_{2}$ : Partial pressure of carbon dioxide; $\mathrm{PaO}_{2}$ : Partial pressure of oxygen; PV+: Positive predictive value; PV—: Negative predictive value; \%pred: Percent predicted; QF: Quadriceps force; ROC: Receiver operating characteristic curves; SARC-F: Strength, assistance in walking, rising from a chair, climbing stairs, and falls; SARC-CalF: SARC-F combined with calf circumference; SMI: Skeletal Muscle Index; $\mathrm{SpO}_{2}$ : Oxygen saturation; SPPB: Short physical performance battery; \%FVC: Percentage of forced vital capacity.
\end{abstract}

\section{Acknowledgements \\ The authors would like to thank all subjects who participated in this study.}

\section{Authors' contributions}

$\mathrm{MH}, \mathrm{NS}, \mathrm{HI}, \mathrm{HT}, \mathrm{HM}$, and RK planned the study. $\mathrm{MH}, \mathrm{HI}, \mathrm{TM}, \mathrm{MO}, \mathrm{HN}$, and RT collected the data. $\mathrm{MH}, \mathrm{NS}, \mathrm{YK}, \mathrm{SS}, \mathrm{SAH}, \mathrm{YI}$, and RK performed the statistical analysis and drafted the manuscript. MH, NS, HI, SAH, YI, HT, HM, and RK participated in the design and coordination of study. All authors read and approved the final manuscript.

\section{Funding}

The study design, collection and analysis of the data, and preparation of the manuscript was not funded by any public, commercial, or not-for-profit sectors.

Availability of data and materials Not applicable. 


\section{Declarations}

\section{Ethics approval and consent to participate}

The subjects gave their written, informed consent. The study was approved by the Human Ethics Review Committee of Nagasaki University Hospital (approval number: 19121610) and was conducted in accordance with the amended Declaration of Helsinki.

\section{Consent for publication}

Not applicable.

\section{Competing interests}

The authors declare that they have no competing interests.

\section{Author details}

'Department of Rehabilitation Medicine, Nagasaki University Hospital, 1-7-1 Sakamoto, Nagasaki 852-8501, Japan. ${ }^{2}$ Department of Physical Therapy Science, Nagasaki University Graduate School of Biomedical Sciences, Nagasaki, Japan. ${ }^{3}$ Department of Respiratory Medicine, Nagasaki University Graduate School of Biomedical Sciences, Nagasaki, Japan. ${ }^{4}$ Clinical Research Center, Nagasaki University Hospital, Nagasaki, Japan. ${ }^{5}$ Institute of Health, Policy, Management and Evaluation, University of Toronto, Toronto, Canada. ${ }^{6}$ Department of Nursing, Nagasaki University Graduate School of Biomedical Sciences, Nagasaki, Japan.

Received: 25 September 2021 Accepted: 24 January 2022

Published online: 25 January 2022

\section{References}

1. Rojas M, Mora AL, Kapetanaki M, Weathington N, Gladwin M, Eickelberg O. Aging and lung disease: clinical impact and cellular and molecular pathways. Ann Am Thorac Soc. 2015;12(12):S222-227.

2. Milne KM, Kwan JM, Guler S, Winstone TA, Le A, Khalil N, Camp PG, Wilcox $P G$, Ryerson CJ. Frailty is common and strongly associated with dyspnoea severity in fibrotic interstitial lung disease. Respirology. 2017;22(4):728-34.

3. Mori H, Tokuda Y. Differences and overlap between sarcopenia and physical frailty in older community-dwelling Japanese. Asia Pac J Clin Nutr. 2019:28(1):157-65.

4. Farooqi MAM, O'Hoski S, Goodwin S, Makhdami N, Aziz A, Cox G, Wald J, Ryerson CJ, Beauchamp MK, Hambly N, et al. Prevalence and prognostic impact of physical frailty in interstitial lung disease: a prospective cohort study. Respirology. 2021. https://doi.org/10.1111/resp.14066.

5. Cruz-Jentoft AJ, Bahat G, Bauer J, Boirie Y, Bruyère $O$, Cederholm T, Cooper C, Landi F, Rolland Y, Sayer AA, et al. Sarcopenia: revised European consensus on definition and diagnosis. Age Ageing. 2019;48(4):601.

6. Cruz-Jentoft AJ, Sayer AA. Sarcopenia. Lancet. 2019;393(10191):2636-46.

7. Xia L, Zhao R, Wan Q, Wu Y, Zhou Y, Wang Y, Cui Y, Shen X, Wu X. Sarcopenia and adverse health-related outcomes: an umbrella review of metaanalyses of observational studies. Cancer Med. 2020;9(21):7964-78.

8. Sasaki Kl, Fukumoto Y. Sarcopenia as a comorbidity of cardiovascular disease. J Cardiol. 2021. https://doi.org/10.1016/j.jjcc.2021.10.013.

9. Kim SE, Kim DJ. Sarcopenia as a prognostic indicator of liver cirrhosis. J Cachexia Sarcopenia Muscle. 2021. https://doi.org/10.1002/jcsm.12869.

10. Patterson KC, Shah RJ, Porteous MK, Christie JD, D'Errico CA, Chadwick M, Triano MJ, Deshpande C, Rossman MD, Litzky LA, et al. Interstitial lung disease in the elderly. Chest. 2017;151(4):838-44.

11. Chen LK, Woo J, Assantachai P, Auyeung TW, Chou MY, lijima K, Jang HC, Kang L, Kim M, Kim S, et al. Asian working group for sarcopenia: 2019 consensus update on sarcopenia diagnosis and treatment. J Am Med Dir Assoc. 2020;21(3):300-307.e302.

12. Malmstrom TK, Morley JE. SARC-F: a simple questionnaire to rapidly diagnose sarcopenia. J Am Med Dir Assoc. 2013;14(8):531-2.

13. Lim JY, Low NA, Merchant RA. Prevalence of sarcopenia in pre-frail community dwelling older adult and utility of SARC-F, SARC-CalF and calf circumference in case finding. J Frailty Sarcopenia Falls. 2020;5(3):53-6.

14. Kera T, Kawai H, Hirano H, Kojima M, Watanabe Y, Motokawa K, Fujiwara Y, Osuka Y, Kojima N, Kim H, et al. Limitations of SARC-F in the diagnosis of sarcopenia in community-dwelling older adults. Arch Gerontol Geriatr. 2020;87:103959.

15. Barbosa-Silva TG, Menezes AM, Bielemann RM, Malmstrom TK, Gonzalez MC. (COCONUT) GdEeCCeN: enhancing SARC-F: improving sarcopenia screening in the clinical practice. J Am Med Dir Assoc. 2016;17(12):1136-41.

16. Raghu G, Remy-Jardin M, Myers JL, Richeldi L, Ryerson CJ, Lederer DJ, Behr J, Cottin V, Danoff SK, Morell F, et al. Diagnosis of idiopathic pulmonary fibrosis: an official ATS/ERS/JRS/ALAT clinical practice guideline. Am J Respir Crit Care Med. 2018;198(5):44-68.

17. Ryerson CJ, Vittinghoff E, Ley B, Lee JS, Mooney JJ, Jones KD, Elicker BM, Wolters PJ, Koth LL, King TE, et al. Predicting survival across chronic interstitial lung disease: the ILD-GAP model. Chest. 2014;145(4):723-8.

18. Yagyu H, Murohashi K, Hara Y, Saigusa Y, Aoki A, Kobayashi N, Kaneko T. Clinical utility of a composite scoring system including Charlson Comorbidity Index score in patients with interstitial lung disease. J Thorac Dis. 2020;12(10):5774-82.

19. Goodman CD, Nijman SFM, Senan S, Nossent EJ, Ryerson CJ, Dhaliwal I, Qu XM, Laba J, Rodrigues GB, Palma DA, et al. A primer on interstitial lung disease and thoracic radiation. J Thorac Oncol. 2020;15(6):902-13.

20. Tanaka S, Kamiya K, Hamazaki N, Matsuzawa R, Nozaki K, Maekawa E, Noda C, Yamaoka-Tojo M, Matsunaga A, Masuda T, et al. Utility of SARC-F for assessing physical function in elderly patients with cardiovascular disease. J Am Med Dir Assoc. 2017;18(2):176-81.

21. Katoh MYH. Test-retest reliability of isometric leg muscle strength measurements made using a hand-held dynamometer restrained by a belt: comparisons during and between sessions. J Phys Ther Sci. 2009;21:239-43.

22. Guralnik JM, Simonsick EM, Ferrucci L, Glynn RJ, Berkman LF, Blazer DG, Scherr PA, Wallace RB. A short physical performance battery assessing lower extremity function: association with self-reported disability and prediction of mortality and nursing home admission. J Gerontol. 1994:49(2):M85-94

23. Dale W, Hemmerich J, Kamm A, Posner MC, Matthews JB, Rothman R, Palakodeti A, Roggin KK. Geriatric assessment improves prediction of surgical outcomes in older adults undergoing pancreaticoduodenectomy: a prospective cohort study. Ann Surg. 2014;259(5):960-5.

24. Nastasi AJ, Bryant TS, Le JT, Schrack J, Ying H, Haugen CE, González Fernández M, Segev DL, McAdams-DeMarco MA. Pre-kidney transplant lower extremity impairment and transplant length of stay: a time-to-discharge analysis of a prospective cohort study. BMC Geriatr. 2018;18(1):246.

25. Yuguchi S, Saitoh M, Oura K, Tahara M, Kamisaka K, Kawamura T, Kato M, Morisawa T, Takahashi T. Impact of preoperative frailty on regaining walking ability in patients after cardiac surgery: multicenter cohort study in Japan. Arch Gerontol Geriatr. 2019;83:204-10.

26. Laboratories ACoPSfCPF. ATS statement: guidelines for the six-minute walk test. Am J Respir Crit Care Med. 2002, 166(1):111-117.

27. Holland AE, Spruit MA, Troosters T, Puhan MA, Pepin V, Saey D, McCormack MC, Carlin BW, Sciurba FC, Pitta F, et al. An official European Respiratory Society/American Thoracic Society technical standard: field walking tests in chronic respiratory disease. Eur Respir J. 2014;44(6):1428-46.

28. Spector WD, Katz S, Murphy JB, Fulton JP. The hierarchical relationship between activities of daily living and instrumental activities of daily living. J Chronic Dis. 1987:40(6):481-9.

29. Patel AS, Siegert RJ, Brignall K, Gordon P, Steer S, Desai SR, Maher TM, Renzoni EA, Wells AU, Higginson IJ, et al. The development and validation of the King's Brief Interstitial Lung Disease (K-BILD) health status questionnaire. Thorax. 2012;67(9):804-10.

30. Mo YH, Zhong J, Dong X, Su YD, Deng WY, Yao XM, Liu BB, Wang $\mathrm{XQ}$, Wang $\mathrm{XH}$. Comparison of three screening methods for sarcopenia in community-dwelling older persons. J Am Med Dir Assoc. 2020;22(4):746-50.

31. da Luz MCL, Pinho CPS, Bezerra GKA, da Conceição Chaves de Lemos M, da Silva Diniz A, Cabral PC. SARC-F and SARC-CalF in screening for sarcopenia in older adults with Parkinson's disease. Exp Gerontol. 2021. 144:111183.

32. Yamada M, Nishiguchi S, Fukutani N, Tanigawa T, Yukutake T, Kayama $\mathrm{H}$, Aoyama T, Arai H. Prevalence of sarcopenia in community-dwelling Japanese older adults. J Am Med Dir Assoc. 2013;14(12):911-5. 
33. Larsson L, Degens H, Li M, Salviati L, Lee YI, Thompson W, Kirkland JL, Sandri M. Sarcopenia: aging-related loss of muscle mass and function. Physiol Rev. 2019;99(1):427-511.

34. Ida S, Murata K, Nakadachi D, Ishihara Y, Imataka K, Uchida A, Monguchi K, Kaneko R, Fujiwara R, Takahashi H. Development of a Japanese version of the SARC-F for diabetic patients: an examination of reliability and validity. Aging Clin Exp Res. 2017;29(5):935-42.

35. Parra-Rodríguez L, Szlejf C, García-González Al, Malmstrom TK, CruzArenas E, Rosas-Carrasco O. Cross-cultural adaptation and validation of the Spanish-language version of the SARC- $F$ to assess sarcopenia in Mexican community-dwelling older adults. J Am Med Dir Assoc. 2016;17(12):1142-6.

36. Kim S, Kim M, Won CW. Validation of the Korean version of the SARC-F Questionnaire to assess sarcopenia: korean frailty and aging cohort study. J Am Med Dir Assoc. 2018;19(1):40-45.e41.

37. Ida S, Kaneko R, Murata K. SARC-F for screening of sarcopenia among older adults: a meta-analysis of screening test accuracy. J Am Med Dir Assoc. 2018;19(8):685-9.

38. Hanada M, Ishimatsu Y, Sakamoto N, Nagura H, Oikawa M, Morimoto Y, Sato S, Mukae H, Kozu R. Corticosteroids are associated with reduced skeletal muscle function in interstitial lung disease patients with mild dyspnea. Respir Med. 2020;174:106184.

39. Yamada Y, Tada M, Mandai K, Hidaka N, Inui K, Nakamura H. Glucocorticoid use is an independent risk factor for developing sarcopenia in patients with rheumatoid arthritis: from the CHIKARA study. Clin Rheumatol. 2020;39(6):1757-64.

\section{Publisher's Note}

Springer Nature remains neutral with regard to jurisdictional claims in published maps and institutional affiliations.

- fast, convenient online submission

- thorough peer review by experienced researchers in your field

- rapid publication on acceptance

- support for research data, including large and complex data types

- gold Open Access which fosters wider collaboration and increased citations

- maximum visibility for your research: over 100M website views per year

At BMC, research is always in progress.

Learn more biomedcentral.com/submissions 